\title{
STABLE SUBALGEBRAS OF LIE ALGEBRAS AND ASSOCIATIVE ALGEBRAS
}

BY

\author{
STANLEY PAGE AND R. W. RICHARDSON, JR.(1)
}

0. Introduction. Let $L=(V, \mu)$ be a Lie (resp. associative) algebra with underlying vector space $V$ and multiplication $\mu$. A subalgebra $S$ of $L$ is stable if $S$ remains a subalgebra under small deformations of $L$. That is, if $L^{\prime}=\left(V, \mu^{\prime}\right)$ is a Lie (resp. associative) algebra with $\mu^{\prime}$ near $\mu$, there exists a subalgebra $S^{\prime}$ of $L^{\prime}$ which is isomorphic to $S$ and whose underlying vector space is near that of $S$. (See $\$ 4$ for a precise definition.) In a letter to one of the authors, J. M. G. Fell has conjectured that if $S$ is a semisimple subalgebra of a finite-dimensional real Lie algebra, then $S$ is stable. The following "stability theorem" shows that this is indeed the case.

Since submitting this paper, we have learned that several of our results for associative algebras, in particular Corollary 11.4(ii), were obtained independently by F. J. Flanigan in his 1966 Dissertation at the University of California, Berkeley.

TheOREM. Let $L=(V, \mu)$ be a finite-dimensional Lie (resp. associative) algebra over either an algebraically closed field or the field $\boldsymbol{R}$ of real numbers. Let $S$ be a subalgebra of $L$ such that $H^{2}(S, V)=0$. Then $S$ is stable.

In the above theorem $V$ is considered as an $S$-module (resp. $S$-bimodule) by means of the adjoint representation of $S$ on $V$ (resp. the multiplication of elements of $V$ by elements of $S) . H^{2}(S, V)$ is the second cohomology space of the Lie (resp. associative) algebra $S$ with coefficients in $V$.

The proof uses only elementary methods, primarily the implicit function theorem, or, in the case of algebraically closed fields, the algebro-geometric analogue thereof.

In the case of a semisimple subalgebra $S$ of the Lie (resp. associative) algebra $L$, a stronger stability theorem holds. Let $P$ be a vector subspace of $L$ which is supplementary to $S$. Then every small deformation of $L$ is equivalent to one in which only the multiplication on $P$ is changed. If, in particular, $S$ is a maximal semisimple subalgebra of $L$, one can take for $P$ the radical of $L$.

1. Preliminaries. By an associative algebra over a field $\boldsymbol{k}$ we mean a vector space $V$ over $\boldsymbol{k}$ together with an associative bilinear multiplication on $V ; A$ is not required to have an identity. An $A$-bimodule is a two-sided representation space for $A$ or, equivalently, a (left) representation space for the algebra $A^{e}=A \otimes_{k} A^{\text {op }}$, where $A^{\text {op }}$ is the opposite algebra of $A$.

Received by the editors May 20, 1966.

(1) The second author acknowledges partial support received from an O.N.R. contract. 
If $L=(V, \mu)$ is a Lie (resp. associative) algebra and $W$ is an $L$-module (resp. $L$ bimodule), then $C(L, W)=\oplus_{n \geq 0} C^{n}(L, W)$ denotes the standard cochain complex for the Lie (resp. associative) algebra $L$ with coefficients in $W ; C^{n}(L, W)$ is the vector space of all alternating $n$-linear (resp. all $n$-linear) maps of $V$ into $W$. $\delta$ denotes the coboundary operator in $C(L, W)$; for the definition of $\delta$ in the case of Lie algebras see [1, p. 282] and in the case of associative algebras see [6]. $H(L, W)=\bigoplus_{n} \geq 0 H^{n}(L, W)$ denotes the corresponding cohomology space; $Z(L, W)=\bigoplus_{n \geqq 0} Z^{n}(L, W)$ denotes the kernel of $\delta$ and $B(L, W)=\bigoplus_{n \geqq 0} B^{n}(L, W)$ the image of $\delta$.

We shall often have occasion to consider simultaneously several distinct cochain complexes $C(L, W)$. We use the same symbol $\delta$ for all coboundary operators involved.

2. Alternating multilinear maps and Lie algebra multiplications. Let $V$ and $W$ be vector spaces over a field $k$. We define $A^{n}(V, W)$ to be the vector space of all alternating $n$-linear maps of $V$ into $W$. We set $A(V, W)=\bigoplus_{n \geqq 0} A^{n}(V, W)$. If $\phi \in A^{n}(V, V)$ and $\psi \in A^{m}(V, V)$, we define $\phi \pi \psi \in A^{n+m-1}(V, V)$, the "hook product" of $\phi$ and $\psi$, by

$$
\phi \pi \psi\left(x_{1}, \ldots, x_{n+m-1}\right)=\sum_{\sigma} \operatorname{sgn}(\sigma) \phi\left(\psi\left(x_{\sigma(1)}, \ldots, x_{\sigma(m)}\right), x_{\sigma(m+1)}, \ldots, x_{\sigma(n+m-1)}\right)
$$

where the sum is taken over all permutations $\sigma$ of $\{1, \ldots, n+m-1\}$ such that $\sigma(1)<\cdots<\sigma(m)$ and $\sigma(m+1)<\cdots<\sigma(n+m-1)$. The hook product determines a bilinear map of $A(V) \times A(V)$ into $A(V)$.

Let $\mu \in A^{2}(V, V)$. Then

$$
\mu \pi \mu(x, y, z)=\mu(\mu(x, y), z)+\mu(\mu(y, z), x)+\mu(\mu(z, x), y) .
$$

Thus $\mu \pi \mu=0$ if and only if $\mu$ satisfies the Jacobi identity, i.e., if and only if $\mu$ is a Lie algebra multiplication on $V$. Thus $M=\left\{\mu \in A^{2}(V, V) \mid \mu \pi \mu=0\right\}$ is precisely the set of Lie algebra multiplications on $V$.

We denote by $G L(V)$ the group of all automorphisms of the vector space $V$. There is a natural representation of $G L(V)$ on each $A^{n}(V, V)$. Precisely, if $g \in G L(V)$ and $\phi \in A^{n}(V, V)$, then

$$
(g \cdot \phi)\left(x_{1}, \ldots, x_{n}\right)=g\left(\phi\left(g^{-1}\left(x_{1}\right), \ldots, g^{-1}\left(x_{n}\right)\right)\right) .
$$

We observe that $M$ is stable under the action of $G L(V)$ on $A^{2}(V, V)$. In fact, let $\mu_{1}, \mu_{2} \in M$ and let $L_{1}=\left(V, \mu_{1}\right)$ and $L_{2}=\left(V, \mu_{2}\right)$ be the corresponding Lie algebras. Then $L_{1}$ and $L_{2}$ are isomorphic if and only if $\mu_{1}$ and $\mu_{2}$ are on the same orbit under the action of $G L(V)$ on $M$.

Let $L=(V, \mu)$ be a Lie algebra and let $W$ be an $L$-module. Then $A(V, W)$ is identical with the cochain complex $C(L, W)$. In particular, the adjoint representation of $L$ defines on $V$ the structure of an $L$-module. The coboundary operator $\delta$ on $C(L, V)=A(V, V)$ can be expressed in terms of the hook product. In fact, if $\phi \in A^{n}(V, V)$, then a simple computation shows that $\delta \phi=(-1)^{n+1} \mu \pi \phi-\phi \pi \mu$. 
3. Multilinear maps and associative algebras. If $V$ and $W$ are vector spaces over a field $\boldsymbol{k}$, we define $L^{n}(V, W)$ to be the vector space of all $n$-linear maps of $V$ into $W$. We set $L(V, W)=\bigoplus_{n \geqq 0} L^{n}(V, W)$. If $\phi \in L^{n}(V, V)$ and $\psi \in L^{m}(V, V)$, then, following Gerstenhaber [5], we define $\phi \circ \psi \in L^{n+m-1}(V, V)$, the "composition product" of $\phi$ and $\psi$, by

$$
\begin{aligned}
\phi \circ \psi\left(x_{1}, \ldots, x_{n+m-1}\right) \\
\quad=\sum_{i=1}^{n}(-1)^{(i+1)(m+1)} \phi\left(x_{1}, \ldots, x_{i-1}, \psi\left(x_{i}, \ldots, x_{i+m-1}\right), x_{i+m}, \ldots, x_{n+m-1}\right) .
\end{aligned}
$$

Let $\mu \in L^{2}(V, V)$. Then $\mu \circ \mu=0$ if and only if $\mu$ defines an associative multiplication on $V$. Thus $M=\left\{\mu \in L^{2}(V, V) \mid \mu \circ \mu=0\right\}$ is precisely the set of associative multiplications on $V$.

There is a canonical representation of $G L(V)$ on each $L^{n}(V, V)$ defined by the formula (2.1). The set $M$ is stable under the action of $G L(V)$ on $L^{2}(V, V)$. Moreover, two points of $M$ lie on the same orbit of $G L(V)$ if and only if the corresponding associative algebras are isomorphic.

Let $A=(V, \mu)$ be an associative algebra and let $W$ be an $A$-bimodule. Then $L(V, W)$ is identical with the cochain complex $C(A, W)$. The multiplication on $A$ defines on $V$ the structure of an $A$-bimodule. Let $\delta$ denote the standard coboundary operator on $C(A, V)=L(V, V)$. If $\phi \in L^{n}(V, V)$, then an easy computation shows that $\delta \phi=(-1)^{n+1} \mu \circ \phi-\phi \circ \mu$.

4. Stable subalgebras. Let $K$ be either an algebraically closed field or the field $\boldsymbol{R}$ of real numbers, let $V$ be a finite-dimensional vector space over $K$ and let $M$ be the algebraic set in $A^{2}(V, V)$ (resp. $L^{2}(V, V)$ ) of all Lie algebra (resp. associative algebra) multiplications on $V$. Let $L=(V, \mu)$ be a Lie (resp. associative) algebra, let $S$ be a subalgebra of $L$ and let $G=G L(V)$; $e$ denotes the identity element of $G$. If $K$ is algebraically closed, all the spaces above are considered as topological spaces supplied with the Zariski topology. If $K=R$, all the above spaces are given the usual Hausdorff topology.

Definition 4.1. $S$ is a stable subalgebra of $L$ if, for every neighborhood $U$ of $e$ in $G$, there exists a neighborhood $U^{\prime}$ of $\mu$ in $M$ such that, to every $\mu^{\prime} \in U^{\prime}$, there corresponds $g \in U$ which satisfies the following condition: the restriction of $g$ to $S$ is an isomorphism of $S$ onto a subalgebra $S^{\prime}$ of $L^{\prime}=\left(V, \mu^{\prime}\right)$.

If $\phi \in C^{2}(L, V)$, we let $\rho(\phi) \in C^{2}(S, V)$ denote the restriction of $\phi$ to $S \times S$. Let $W$ be a supplementary subspace of $S$ in $V$. We let $\tau(\phi)$ denote the restriction of $\phi$ to the union of the three sets $S \times S, S \times W$ and $W \times S$. Let

$$
N=\{(g, m) \in G \times M \mid \rho(g \cdot m)=\rho(\mu)\} .
$$

Let $\operatorname{pr}_{M}: G \times M \rightarrow M$ be the projection map. Then $m \in \operatorname{pr}_{M}(N)$ if and only if the Lie algebra $L^{\prime}=(V, m)$ has a subalgebra which is isomorphic to $S$. We let

$$
N_{1}=\{(g, m) \in G \times M \mid \tau(g \cdot m)=\tau(\mu)\} .
$$


Let $\pi: N \rightarrow M$ (resp. $\pi_{1}: N_{1} \rightarrow M$ ) denote the restriction to $N$ (resp. $N_{1}$ ) of the projection $\operatorname{pr}_{\mathrm{M}}$. We shall consider the following conditions on the subalgebra $S$ of $L$ :

CoNDITION (a). $\pi$ maps every neighborhood of $(e, \mu)$ in $N$ onto a neighborhood of $\mu$ in $M$.

CoNDITION (b). $\pi_{1}$ maps every neighborhood of $(e, \mu)$ in $N_{1}$ onto a neighborhood of $\mu$ in $M$.

If $K=R$, we consider these stronger conditions on $S$ :

Condition ( $\left.\mathrm{a}^{\prime}\right)$ (resp. Condition ( $\left.\mathrm{b}^{\prime}\right)$ ). There exists an open neighborhood $U$ of $\mu$ in $M$ and a real-analytic map $\sigma: U \rightarrow G$ with $\sigma(\mu)=e$ such that $(\sigma(m), m) \in N$ (resp. $\left.(\sigma(m), m) \in N_{1}\right)$ for every $m \in U$.

It is elementary to show that each of the four conditions above implies that $S$ is stable.

Conditions (b) and (b') imply that if $L_{1}=\left(V, \mu_{1}\right)$ is a Lie (resp. associative) algebra with $\mu_{1}$ sufficiently near $\mu$, then $L_{1}$ is isomorphic to a Lie algebra $L_{2}=\left(V, \mu_{2}\right)$ with the following property: if $s \in S$ and $x \in V$, then $\mu(s, x)=\mu_{2}(s, x)$ and $\mu(x, s)=\mu_{2}(x, s)$.

5. Differential geometric preliminaries. For the basic facts concerning realanalytic manifolds, we refer to [7]. If $X$ is a real-analytic manifold and $x \in X$, we denote by $T(X, x)$ the tangent space of $X$ at $x$. If $Y$ is a submanifold of $X$ and $y \in Y$, we identify $T(Y, y)$ with a subspace of $T(X, y)$. If $X$ is a real vector space and $x \in X$, we identify $T(X, x)$ with $X$. If $X$ and $Y$ are real-analytic manifolds and $f: X \rightarrow Y$ is a real-analytic map, then $d f_{x}: T(X, x) \rightarrow T(Y, f(x))$ denotes the differential of $f$ at $x \in X$.

We will use the following version of the implicit function theorem and inverse function theorem:

5.1. Let $X$ and $Y$ be real-analytic manifolds and let $f: X \rightarrow Y$ be a real-analytic map. Let $x \in X$ be such that, setting $y=f(x)$, the differential $d f_{x}: T(X, x) \rightarrow T(Y, y)$ is surjective. Then if $U$ is a neighborhood of $x$ in $X, f(U)$ is a neighborhood of $y$ in $Y$. Moreover, there exists an open neighborhood $U_{0}$ of $x$ in $X$ such that the following conditions hold: (a) $Z=f^{-1}(y) \cap U_{0}$ is a closed (real-analytic) submanifold of $U_{0}$ and $T(Z, x)=$ kernel $\left(d f_{x}\right)$; (b) there exists a closed submanifold $W$ of $U_{0}$ with $x \in W$ such that $f(W)$ is an open neighborhood of $y$ in $Y$ and $f$ induces a real-analytic isomorphism of $W$ and $f(W)$.

5.1 is equivalent to the usual local formulation of the implicit and inverse function theorems in the real-analytic case as given, for example, in [4, pp. 265-269].

\section{The stability theorem for real Lie algebras.}

THEOREM 6.1. Let $L=(V, \mu)$ be a finite-dimensional real Lie algebra and let $S$ be a subalgebra of $L$ such that $H^{2}(S, V)=0$. Then $S$ satisfies condition $\left(\mathrm{a}^{\prime}\right)$. In particular, $S$ is a stable subalgebra. 
The proof of Theorem 6.1 will occupy the rest of this section.

We let $M$ denote the real-algebraic set in $A^{2}(V, V)$ of all Lie algebra multiplications on $V$ and let $G=G L(V)$. We observe that $C(L, V)=A(V, V)$ and $C(S, V)=A(S, V)$. (We identify $S$ with its underlying subspace.) If $\phi \in A^{n}(V, V)$, we let $\rho(\phi)$ denote the restriction of $\phi$ to $S \times \cdots \times S$. $\rho$ is a surjective linear map of $A^{n}(V, V)$ onto $A^{n}(S, V)$ and $\rho \circ \delta=\delta \circ \rho$.

The proof of 6.1 will require three distinct applications of the implicit and inverse function theorems. Since $\mu$ is not necessarily a simple point of $M$ (i.e., $M$ may not be locally a manifold at $\mu$ ), it is necessary to replace $M$ by a larger realalgebraic set $M_{1}$ which has $\mu$ as a simple point. This motivates the following result, which is stated more generally than needed in the proof of 6.1 since it will also be used in a later section.

6.2. Let $C$ be a vector subspace of $A^{2}(V, V)$ which contains $\dot{Z}^{2}(L, V)$, let $C_{0}=\delta(C)$, let $C_{1}$ be supplementary to $C_{0}$ in $B^{3}(L, V)$ and let $C_{2}$ be supplementary to $B^{3}(L, V)$ in $A^{3}(V, V)$. Let $\pi_{1}: A^{3}(V, V) \rightarrow C_{1}$ be the projection with kernel $C_{0}+C_{2}$ and let $M_{1}=\left\{\phi \in A^{2}(V, V) \mid \pi_{1}(\phi \pi \phi)=0\right\}$. Then $M \subset M_{1}$ and there exists an open neighborhood $U_{1}$ of $\mu$ in $A^{2}(V, V)$ such that $M^{\prime}=U_{1} \cap M_{1}$ is a closed (real-analytic) submanifold of $U_{1}$ and $T\left(M^{\prime}, \mu\right)=C$.

Proof. It is obvious that $M \subset M_{1}$. Let $f: A^{2}(V, V) \rightarrow A^{3}(V, V)$ be defined by $f(\phi)=\phi \pi \phi ; f$ is a polynomial mapping. We have

$$
f(\mu+\phi)=\mu \pi \phi+\phi \pi \mu+\phi \pi \phi=-\delta \phi+\phi \pi \phi .
$$

It follows that $d f_{\mu}=-\delta$. Let $F: A^{2}(V, V) \rightarrow C_{1}$ be the composite map $\pi_{1} \circ f$. Since $\pi_{1}$ is linear, $d F_{\mu}=-\pi_{1} \circ \delta$. Hence $d F_{\mu}$ is surjective and $C=\operatorname{kernel}\left(d F_{\mu}\right)$. The proof of 6.2 now follows from 5.1.

6.3. Let $C=\rho^{-1}\left(Z^{2}(S, V)\right)$ and let $M^{\prime}$ be as in 6.2. Let $\gamma: G \rightarrow A^{2}(V, V)$ be defined by $\gamma(g)=g \cdot \mu$. It follows from (2.1) that $d \gamma_{e}(\psi)=\psi \pi \mu-\mu \pi \psi=-\delta \psi$. Thus $d \gamma_{e}=-\delta$.

Let $\eta: G \times M^{\prime} \rightarrow A^{2}(V, V)$ be defined by $\eta(g, m)=g \cdot m$. We note that

$$
T\left(G \times M^{\prime},(e, \mu)\right)=A^{1}(V, V) \times C .
$$

It follows from the above computation of $d \gamma_{e}$ that $d \eta_{(e, \mu)}(\psi, \phi)=-\delta \psi+\phi$.

Let $E$ be a supplementary subspace to $C$ in $A^{2}(V, V)$ and let $E^{\prime}=\rho(E)$. We observe that $E^{\prime} \cap Z^{2}(S, V)=\{0\}$. We thus have a direct sum decomposition $A^{2}(S, V)=Z^{2}(S, V)+E^{\prime}$. Let $\pi_{Z}: A^{2}(S, V) \rightarrow Z^{2}(S, V)$ and $\pi_{E^{\prime}}: A^{2}(S, V) \rightarrow E^{\prime}$ be the corresponding projections. Let $\beta: G \times M^{\prime} \rightarrow Z^{2}(S, V)$ be the composite map $\pi_{Z} \circ \rho \circ \eta$; note that $\beta(e, \mu)=\rho(\mu)$. Since $\pi_{z}$ and $\rho$ are linear maps, we have $d \beta_{(e, \mu)}=\pi_{Z} \circ \rho \circ d \eta_{(e, \mu)}$. Thus $d \beta_{(e, \mu)}(\psi, \phi)=-\delta(\rho(\psi))+\pi_{Z}(\rho(\phi))$. Since $T\left(M^{\prime}, \mu\right)=C$ and $\rho(C)=Z^{2}(S, V)$, it follows that $d \beta_{(e, \mu)}$ is surjective. Hence the implicit function theorem applies and there exists an open neighborhood $U_{2}$ of $(e, \mu)$ in $G \times M^{\prime}$ such that $N^{\prime}=U_{2} \cap \beta^{-1}(\rho(\mu))$ is a closed submanifold of $U_{2}$ and $T\left(N^{\prime},(e, \mu)\right)$ $=\operatorname{kernel}\left(d \beta_{(e, \mu)}\right)$. 
Let $\pi: N^{\prime} \rightarrow M^{\prime}$ denote the restriction to $N^{\prime}$ of the projection

$$
\operatorname{pr}_{M^{\prime}}: G \times M^{\prime} \rightarrow M^{\prime} \text {. }
$$

6.4. If $H^{2}(S, V)=0$, then the differential $d \pi_{(e, \mu)}: T\left(N^{\prime},(e, \mu)\right) \rightarrow T\left(M^{\prime}, \mu\right)$ is surjective.

Proof. Let $\phi \in T\left(M^{\prime}, \mu\right)=C$. By definition of $C$ we have $\rho(\phi) \in Z^{2}(S, V)$ $=B^{2}(S, V)$. Thus there exists $\theta \in A^{\prime}(S, V)$ such that $\delta \theta=\rho(\phi)$. Since $\rho$ is surjective, there exists $\psi \in A^{1}(V, V)$ such that $\rho(\psi)=\theta$; therefore $\delta(\rho(\psi))=\rho(\phi)=\pi_{z}(\rho(\phi))$. Consequently

$$
d \beta_{(e, \mu)}(\psi, \phi)=-\delta(\rho(\psi))+\pi_{z}(\rho(\phi))=0 .
$$

It follows that $(\psi, \phi) \in T\left(N^{\prime} m(e, \mu)\right)$. Since $d \pi_{(e, \mu)}(\psi, \phi)=\phi$, this completes the proof of 6.4.

If $(g, m) \in N^{\prime}$, then $\pi_{Z}(\rho(g, m))=\rho(\mu)$ but it is not necessarily true that $\rho(g, m)=\rho(\mu)$. However, the following lemma shows that this $i s$.the case if $m \in M$ (i.e., if $m$ is a Lie algebra multiplication) and if $(g, m)$ is sufficiently near $(e, \mu)$.

LeMma 6.5. There exists a neighborhood $U_{3}$ of $(e, \mu)$ in $G \times M$ such that, if $(g, m) \in U_{3}$ and if $\pi_{z}(\rho(g \cdot m))=\rho(\mu)$, then $\rho(g \cdot m)=\rho(\mu)$.

Proof. Let $(g, m) \in G \times M$ with $\pi_{z}(\rho(g \cdot m))=\rho(\mu)$. Then $g \cdot m=\mu+a+b$ with $a \in \operatorname{kernel}(\rho)$ and $b \in E$. We recall that the restriction of $\delta \circ \rho$ to $E$ is a monomorphism. We have

$$
\begin{aligned}
0 & =(g \cdot m) \pi(g \cdot m)=(\mu+a+b) \pi(\mu+a+b) \\
& =-\delta a-\delta b+a \pi a+b \pi b+a \pi b+b \pi a .
\end{aligned}
$$

We note that $\rho(a \pi a)=0=\rho(\delta a)$. If we apply $\rho$ to the above equation we obtain

$$
0=\delta(\rho(b))+\rho((a+b) \pi b)+\rho(b \pi a) .
$$

Let $\pi_{C}: A^{2}(V, V) \rightarrow C$ be the projection with kernel $E$. We note that $\pi_{C}(a+b)=a$. Thus the above equation may be rewritten

$$
0=\delta(\rho(b))+\rho((a+b) \pi b)+\rho\left(b \pi \pi_{C}(a+b)\right) .
$$

For each $x \in A^{2}(V, V)$, let $\lambda_{x}: E \rightarrow A^{3}(S, V)$ be the linear map defined by

$$
\lambda_{x}(\phi)=\delta(\rho(\phi))+\rho(x \pi \phi)+\rho\left(\phi \pi \pi_{C}(x)\right) .
$$

Then $\lambda_{0}=\delta \circ \rho$ is a monomorphism. Thus there exists a neighborhood $J$ of 0 in $A^{2}(V, V)$ such that $\lambda_{x}$ is a monomorphism for every $x \in J$. Choose $U_{3}$, a neighborhood of $(e, \mu)$ in $G \times M$, such that, if $\left(g^{\prime}, m^{\prime}\right) \in U_{3}$, then $\left(g^{\prime} \cdot m^{\prime}-\mu\right) \in J$. Assume now that $(g, m) \in U_{3}$. We have $g \cdot m=\mu+a+b$ and hence $x=(a+b) \in J$. Then (6.5.1) shows that $\lambda_{x}(b)=0$. Since $\lambda_{x}$ is a monomorphism, it follows that $b=0$. Thus $g \cdot m=\mu+a$ and hence $\rho(g \cdot m)=\rho(\mu)$. This proves 6.5 .

6.6. It follows from 6.4 and 5.1(b) that there exist an open neighborhood $U^{\prime}$ of $\mu$ in $M^{\prime}$ and a real-analytic map $h: U^{\prime} \rightarrow N^{\prime}$ such that $h(\mu)=(e, \mu)$ and $\pi \circ h$ is the identity map of $U^{\prime}$. We may assume $h\left(U^{\prime}\right) \subset U_{3}$. We may write $h(m)=(s(m), m)$ 
where $s: U^{\prime} \rightarrow G$ is a real-analytic map. Let $N=\{(g, m) \in G \times M \mid \rho(g \cdot m)=\rho(\mu)\}$, let $U=M \cap U^{\prime}$ and let $\sigma$ denote the restriction of $s$ to $U$. Then 6.5 shows that $(\sigma(m), m) \in N$ if $m \in U$. Thus $S$ satisfies Condition (a'). This completes the proof of Theorem 6.1.

\section{The stability theorem for real associative algebras.}

THEOREM 7.1. Let $A=(V, \mu)$ be a finite-dimensional associative algebra over $\boldsymbol{R}$ and let $S$ be a subalgebra of $A$ such that $H^{2}(S, V)=0$. Then $S$ satisfies Condition ( $\left.a^{\prime}\right)$ and hence is a stable subalgebra of $A$.

The proof of Theorem 7.1 is the same as that of Theorem 6.1 if we consistently replace $A^{n}(V, V)$ and $A^{n}(S, V)$ by $L^{n}(V, V)$ and $L^{n}(S, V)$ and replace the hook product of alternating multilinear maps by the composition product of multilinear maps. We omit further details.

8. Preliminary algebraic geometry. Our basic reference for algebraic geometry is [2]; we shall follow the terminology used therein. In particular, we consider algebraic varieties (i.e., the irreducible case) over an algebraically closed field $K$. An algebraic variety $X$ is considered as a topological space with the Zariski topology. If $x \in X$, we denote by $T(X, x)$ the tangent space of $X$ at $x$. If $Y$ is a subvariety of $X$ and $x \in Y$, we shall identify $T(Y, x)$ with a subspace of $T(X, x)$ in the usual manner. The point $x \in X$ is simple if $\operatorname{dim} T(X, x)=\operatorname{dim} X$. If $f: X \rightarrow Y$ is a morphism of algebraic varieties, and $x \in X$, we denote by

$$
d f_{x}: T(X, x) \rightarrow T(Y, f(x))
$$

the differential of $f$ at $x$ ( $d f_{x}$ is called the derived mapping of $f$ at $x$ in [2]).

We have the following algebro-geometric analogue of 5.1:

Proposition 8.1. Let $f: X \rightarrow Y$ be a morphism of algebraic varieties, let $x \in X$ and let $y=f(x)$. Suppose that $x$ (resp. $y$ ) is a simple point of $X$ (resp. Y) and that the differential $d f_{x}: T(X, x) \rightarrow T(Y, y)$ is a surjective mapping.

(a) If $U$ is a neighborhood of $x$ in $X$, then $f(U)$ is a neighborhood of $y$ in $Y$.

(b) There is exactly one irreducible component $X_{1}$ of $f^{-1}(y)$ which contains $x$. Moreover, $x$ is a simple point of $X_{1}$ and $T\left(X_{1}, x\right)=$ kernel $\left(d f_{x}\right)$.

Proof. The proof of (b) can easily be reduced to the case in which $X$ and $Y$ are affine varieties. In this case, it follows from [8, p. 354, Proposition 1] by a straightforward argument. The condition on $d f_{x}$ implies that $f$ is dominant. Using (b), the proof of (a) now follows from [2, p. 195, Proposition 3].

\section{Stability for algebras over algebraically closed fields.}

THEOREM 9.1. Let $L=(V, \mu)$ be a finite-dimensional Lie (resp. associative) algebra over an algebraically closed field and let $S$ be a subalgebra of $L$ such that $H^{2}(S, V)=0$. Then $S$ satisfies Condition (a) and hence is stable. 
We give the proof only for the case of Lie algebras. Our proof closely parallels that of Theorem 6.1. We shall often follow the notation of $\$ 6$ without explicit reference.

Let $C=\rho^{-1}\left(Z^{2}(S, V)\right)$ and let $M_{1}$ be defined as in 6.2. The proof of 6.2 combined with 8.1 shows that there is precisely one irreducible component $M^{\prime}$ of $M_{1}$ which contains $\mu$. Moreover, $\mu$ is a simple point of $M^{\prime}$ and $T\left(M^{\prime}, \mu\right)=C$. Let $M_{0}$ denote the union of the irreducible components of $M$ which contain $\mu$. It follows easily that $M_{0} \subset M^{\prime}$. Let the morphism $\beta: G \times M^{\prime} \rightarrow Z^{2}(S, V)$ be defined as in 6.3. Then the argument given there shows that $d \beta_{(e, \mu)}(\psi, \phi)=-\delta(\rho(\psi))+\pi_{z}(\rho(\phi))$. In particular, $d \beta_{(e, \mu)}$ is surjective. By 8.1 there exists exactly one irreducible component $M^{\prime}$ of $\beta^{-1}(\rho(\mu))$ containing $(e, \mu),(e, \mu)$ is a simple point of $N^{\prime}$ and

$$
T\left(N^{\prime},(e, \mu)\right)=\operatorname{kernel}\left(d \beta_{(e, \mu)}\right) \text {. }
$$

The statements and proof of 6.4 and 6.5 are also valid in this case, with the obvious modifications. It is easy to see that these two results, combined with 8.1, imply that $S$ satisfies Condition (a). This proves 9.1 .

10. An algebraic digression. The results of this section will be used to show that semisimple subalgebras of Lie (resp. associative) algebras satisfy Condition (b).

Let $L=(V, \mu)$ be a Lie algebra over a field $k$ and let $S$ be a subalgebra of $L$; we identify $S$ with its underlying vector space. Let $V^{n}$ denote the $n$-fold Cartesian product $V$ and let $F_{n}$ denote the subset of $V^{n}$ consisting of all $\left(x_{1}, \ldots, x_{n}\right)$ satisfying the following condition: There exists at most one index $i$ such that $x_{i} \notin S$. Let $P$ be a vector space over $\boldsymbol{k}$. A map $\phi: F_{n} \rightarrow P$ is said to be multilinear (resp. alternating) if $\phi$ is the restriction of a multilinear (resp. alternating) map $\phi^{\prime}: V^{n} \rightarrow P$ (this definition works only for vector spaces). Let, now, $P$ be an $L$-module. We define $F^{n}(L, S, P)$ to be the vector space of all alternating multilinear maps of $F_{n}$ into $P$. We set $F(L, S, P)=\oplus_{n \geqq 0} F^{n}(L, S, P)$. The coboundary operator $\delta: F(L, S, P) \rightarrow F(L, S, P)$ is defined by the usual formula (see, e.g., [1, p. 282]). One checks that $\delta$ is well defined in this case. $\delta$ is homogeneous of degree 1 and $\delta \circ \delta=0$. Thus $(F(L, S, P), \delta)$ is a cochain complex. We denote by $E(L, S, P)$ $=\bigoplus_{n \geqq 0} E^{n}(L, S, P)$ the corresponding cohomology space. In particular, the adjoint representation of $L$ defines an $L$-module structure on $V$ and we can consider the cohomology space $E(L, S, V)$.

If $R$ is a Lie (resp. associative) algebra then an exact sequence

$$
0 \longrightarrow M \stackrel{i}{\longrightarrow} N \stackrel{j}{\longrightarrow} P \longrightarrow 0
$$

of $R$-modules is, by definition, an extension of $P$ by $M$. The extension splits if there exists an $R$-module homomorphism $\eta: P \rightarrow N$ such that $j \circ \eta=1_{P}$.

The adjoint representation of $S$ on $V$ defines $V$ as an $S$-module. $S$ is a submodule and thus $V / S$ inherits an $S$-module structure. There is thus an induced $S$-module structure on $\operatorname{Hom}_{k}(V / S, V / S)$. 
Proposition 10.1. Let $L=(V, \mu)$ be a Lie algebra and let $S$ be a subalgebra of $L$ which satisfies the following conditions:

(a) $H^{2}(S, V)=0$.

(b) $H^{1}\left(S, \operatorname{Hom}_{k}(V / S, V / S)\right)=0$.

(c) Every extension of the $S$-module $V / S$ by the $S$-module $S$ splits. Then $E^{2}(L, S, V)=0$.

Proof. We sketch the proof. Let $W$ be a supplementary subspace of $S$ in $V$ and let $\pi_{W}: V \rightarrow W$ be the projection with kernel $S$. Let $\phi \in F^{2}(L, S, V)$ with $\delta \phi=0$. It follows from (a) that there exists $\psi \in F^{1}(L, S, V)$ such that $\phi_{1}=\phi-\delta \psi$ vanishes on $S \times S$. A straightforward argument using (b) shows that there exists $\psi_{1} \in F^{1}(L, S, V)$ such that $\phi_{2}=\phi_{1}-\delta \psi_{1}$ satisfies the following conditions: $\phi_{2}$ vanishes on $S \times S$ and $\pi_{W} \circ \phi_{2}$ vanishes on $S \times W$. Finally, (c) implies the existence of $\psi_{2} \in F^{1}(L, S, V)$ such that $\delta \psi_{2}=\phi_{2}$. Hence, $\phi=\delta\left(\psi+\psi_{1}+\psi_{2}\right)$. This completes the proof.

COROLlARY 10.2. Let $L=(V, \mu)$ be a finite-dimensional Lie algebra over a field of characteristic 0 and let $S$ be a semisimple subalgebra of $L$. Then $E^{2}(L, S, V)=0$.

Proof. It is shown in [3] that a semisimple Lie algebra $S$ satisfies (a), (b), and (c) of 10.1.

Similar considerations apply to the case of associative algebras. Let $A=(V, \mu)$ be an associative algebra and let $S$ be a subalgebra of $A . F_{n}$ is defined as above. If $P$ is an $A$-bimodule, we define $F^{n}(A, S, P)$ to be the vector space of all multilinear maps of $F_{n}$ into $P$. We define $F(A, S, P)=\oplus_{n \geqq 0} F^{n}(A, S, P)$. The coboundary operator $\delta: F(A, S, P) \rightarrow F(A, S, P)$ is defined by the usual formula (see [6]; one checks that $\delta$ is well defined in this case). $\delta$ is homogeneous of degree 1 and $\delta \circ \delta=0$, thus $(F(A, S, P), \delta)$ is a cochain complex. We denote by $E(A, S, P)$ $=\bigoplus_{n \geqq 0} E^{n}(A, S, P)$ the corresponding cohomology space. In particular, $V$ has a natural structure of $A$-bimodule and we can consider $E(A, S, V)$.

Let $S^{\text {op }}$ denote the opposite algebra of $S$ and let $S^{e}=S \otimes_{k} S^{\text {op }}$. Then $V / S$ has a natural structure of an $S$-bimodule, that is, of an $S^{e}$-module. Thus there is an induced structure of $S^{e}$-module on $\operatorname{Hom}_{k}(V / S, V / S)$.

Proposition 10.3. Let $A=(V, \mu)$ be an associative algebra and let $S$ be a subalgebra of $A$ which satisfies the following conditions:

(a) $H^{2}(S, V)=0$.

(b) $H^{1}\left(S^{e}, \operatorname{Hom}_{k}(V / S, V / S)\right)=0$.

(c) Every extension of the $S^{e}$-module $V / S$ by the $S^{e}$-module $S$ splits. Then $E^{2}(L, S, V)=0$.

The proof is similar to that of Proposition 10.1 although somewhat more complicated. We omit the details.

COROLlARY 10.4. Let $A$ be a finite-dimensional associative algebra over a field and let $S$ be a separable subalgebra of $A$. Then $E^{2}(A, S, V)=0$. 
The proof follows from the fact that a separable subalgebra satisfies conditions (a), (b), and (c) above.

\section{A strengthened stability theorem.}

THEOREM 11.1. Let $L=(V, \mu)$ be a finite-dimensional Lie (resp. associative) algebra over a field $K$ and let $S$ be a subalgebra of $L$ such that $E^{2}(L, S, V)=0$. (i) If $K$ is algebraically closed, then $S$ satisfies condition (b). (ii) If $K=\boldsymbol{R}$, then $S$ satisfies condition $\left(\mathrm{b}^{\prime}\right)$.

We shall give the proof only for the case of Lie algebras over an algebraically closed field. The proofs in the other cases are similar. Our proof parallels those of Theorems 6.1 and 8.1.

If $\phi \in A^{n}(V, V)$, let $\tau(\phi)$ denote the restriction of $\phi$ to $F_{n}$. Let

$$
Z^{2}=\left\{\theta \in F^{2}(L, S, V) \mid \delta \theta=0\right\}
$$

and let $C=\left\{\phi \in A^{2}(V, V) \mid \tau(\phi) \in Z^{2}\right\}$. Then $Z^{2}(L, V) \subset C$. Let $M_{1}$ be defined as in 6.2. Then the proof of 6.2 , combined with 8.1 , shows that there is exactly one irreducible component $M^{\prime}$ of $M_{1}$ which contains $\mu$. Moreover, $\mu$ is a simple point of $M^{\prime}$ and $T\left(M^{\prime}, \mu\right)=C$.

Let $E$ be a supplementary subspace of $C$ in $A^{2}(V, V)$ and let $E^{\prime}=\tau(E)$. We have a direct sum decomposition $F^{2}(L, S, V)=Z^{2}+E^{\prime}$. Let $\pi_{Z}: F^{2}(L, S, V) \rightarrow Z^{2}$ be the projection with kernel $E^{\prime}$. Let $\eta: G \times M^{\prime} \rightarrow A^{2}(V, V)$ be defined by $\eta(g, m)$ $=g \cdot m$ and let $\beta: G \times M^{\prime} \rightarrow Z^{2}$ be the composite map $\pi_{z} \circ \tau \circ \eta$. Essentially the same argument given in 6.3 shows that $d \beta_{(e, \mu)}$ is surjective. Thus, by 8.1 , there exists precisely one irreducible component $N^{\prime}$ of $\beta^{-1}(\tau(\mu))$ containing $(e, \mu),(e, \mu)$ is a simple point of $N^{\prime}$ and $T\left(N^{\prime},(e, \mu)\right)=\operatorname{kernel}\left(d \beta_{(e, \mu)}\right)$.

Let $\pi: N^{\prime} \rightarrow M^{\prime}$ denote the restriction to $N^{\prime}$ of the projection

$$
\operatorname{pr}_{M^{\prime}}: G \times M^{\prime} \rightarrow M^{\prime}
$$

11.2. If $E^{2}(L, S, V)=0$, then the differential $d \pi_{(e, \mu)}: T\left(N^{\prime},(e, \mu)\right) \rightarrow T\left(M^{\prime}, \mu\right)$ is surjective.

The proof of 11.2 is similar to that of 6.4.

11.3. There exists a neighborhood $U_{3}$ of $(e, \mu)$ in $G \times M$ such that, if $(g, m) \in U_{3}$ and if $\pi_{z}(\tau(g \cdot m))=\tau(\mu)$, then $\tau(g \cdot m)=\tau(\mu)$.

The proof of 11.3 is similar to that of 6.5.

The proof of 11.1 is a straightforward consequence of $11.2,11.3$, and 8.1 . We omit further details.

COROLLARY 11.4. (i) Let $L$ be a finite-dimensional Lie algebra over an algebraically closed field of characteristic $\mathbf{0}$ (resp. over $\boldsymbol{R}$ ) and let $S$ be a semisimple subalgebra of $L$. Then $S$ satisfies Condition (b) (resp. (b')). (ii) Let $A$ be a finite-dimensional associative algebra over an algebraically closed field (resp. over $R$ ) and let $S$ be a semisimple subalgebra of $L$. Then $S$ satisfies Condition (b) (resp. $\left.\left(b^{\prime}\right)\right)$. 
11.4 follows immediately from $11.1,10.2$, and 10.4 . We remark that, if the base field is algebraically closed or of characteristic 0 , semisimple subalgebras of an associative algebra are separable.

\section{REFERENCES}

1. H. Cartan and S. Eilenberg, Homological algebra, Princeton Univ. Press, Princeton, N. J., 1956.

2. C. Chevalley, Fondements de la géométrie algébrique, Course notes at the Institut Henri Poincaré, Paris, 1958.

3. C. Chevalley and S. Eilenberg, Cohomology theory of Lie groups and Lie algebras, Trans. Amer. Math. Soc. 63 (1948), 85-124.

4. J. Dieudonné, Foundations of modern analysis, Academic Press, New York, 1960.

5. M. Gerstenhaber, The cohomology structure of an associative ring, Ann. of Math. (2) 78 46 (1963), 267-289.

6. G. Hochschild, On the cohomology groups of an associative algebra, Ann. of Math. (2) (1945), 58-67.

7. - The structure of Lie groups, Holden-Day, San Francisco, Calif., 1965.

8. A. Weil, Foundations of algebraic geometry, rev. ed., Colloq. Publ., Vol. 29, Amer. Math. Soc., Providence, R. I., 1962.

UNIVERSITY OF WASHINGTON, SeATtLe, Washington 